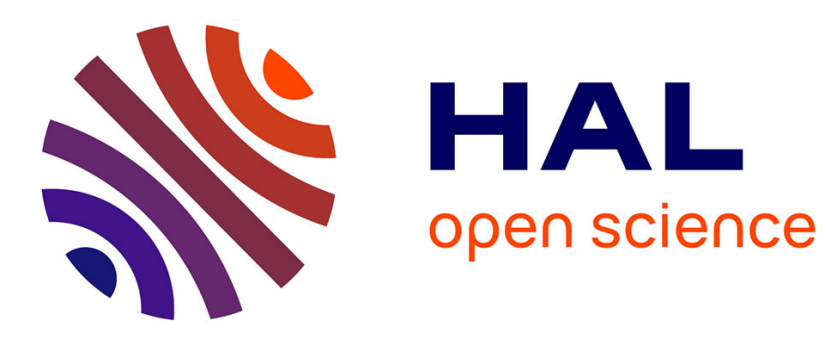

\title{
Multiscale statistical signal processing
}

Michèle Basseville, Albert Benveniste

\section{To cite this version:}

Michèle Basseville, Albert Benveniste. Multiscale statistical signal processing. [Research Report] RR-0970, INRIA. 1989. inria-00075589

\section{HAL Id: inria-00075589 \\ https://hal.inria.fr/inria-00075589}

Submitted on 24 May 2006

HAL is a multi-disciplinary open access archive for the deposit and dissemination of scientific research documents, whether they are published or not. The documents may come from teaching and research institutions in France or abroad, or from public or private research centers.
L'archive ouverte pluridisciplinaire HAL, est destinée au dépôt et à la diffusion de documents scientifiques de niveau recherche, publiés ou non, émanant des établissements d'enseignement et de recherche français ou étrangers, des laboratoires publics ou privés. 


\title{
Rapports de Recherche
}

\author{
$\mathbf{N}^{\circ} 970$

\section{Programme 5}

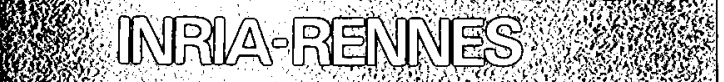

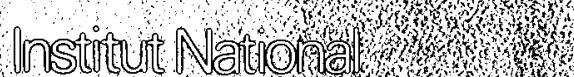

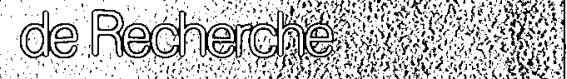
en brormanglon?

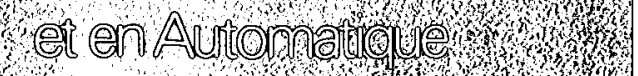
Y

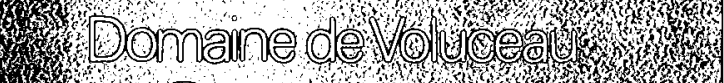

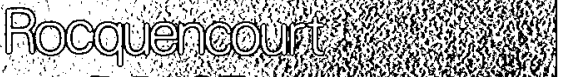

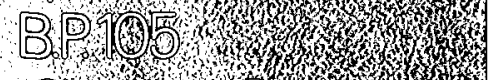

资

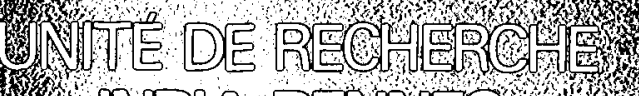
3

\section{MULTISCALE STATISTICAL SIGNAL PROCESSING}

Michèle BASSEVILLE Albert BENVENISTE 
Campus Universitaire de Beaulieu 35042-RENNES CÉDEX FRANCE

Téléphone : 99362000

Télex: UNIRISA $950473 \mathrm{~F}$

Télécopie: 99383832

\title{
Multiscale Statistical Signal Processing *
}

\author{
Publication Interne $\mathrm{n}^{\circ} 445$ - 16 Pages - Décembre 1988 \\ MiChÈle BASSEVILle (IRISA/CNRS) \\ Albert BenVEniste (IRISA/INRIA)
}

\begin{abstract}
In many applications (e.g. recognition of geophysical and biomedical signals and multiscale analysis of images), it is of interest to analyse and recognize phenomena occurring at different scales. The recently introduced wavelet transforms provide a time-and-scale decomposition of signals. But no corresponding statistical framework is currently available. Our purpose is to fill this gap. Our starting point is the theory of stationary processes on homogeneous trees which is shown to be the convenient framework for such a statistical multiscale signal processing. Here we introduce the corresponding system theory by investigating $A R$ processes. This paper is a very short abstract of the report [7] intended to promote a widespread use of french.
\end{abstract}

\section{Modèles Statistiques Temps-Echelle en Traitement du Signal}

\section{Résumé}

Dans de nombreuses applications (par exemple reconnaissance de signaux géophysiques ou biomédicaux, analyse pyramidale d'images), il est intéssant d'analyser et de reconnaitre des phénomènes se produisant à différentes échelles. Les transformées en ondelettes récemment introduites fournissent une décomposition de signaux en temp et en échelle. Mais à l'heure actuelle il n'existe pas de contexte statistique correspondant. Notre but est de combler cette lacune. Notre point de départ est la théorie des processus stationnaires sur les arbres homogènes, dont on montre qu'il est le contexte adéquat pour un tel traitement statistique du signal multi-échelle. Nous introduisons ici la théorie des systèmes correspondante en étudiant le problème de factorisation spectrale et les processus AR.

*Ce travail est réalisé dans le cadre d'une collaboration avec le MIT/LIDS. Nous remercions Alan Willsky et Ken Chou pour les discussions fructueuses que nous avons eues pendant leur séjour à l'IRISA. Nous remercions aussi Jean-Pierre Conze, Yves Guivarch et Albert Raugi du laboratoire de Probabilités de l'IRMAR (Rennes) de nous avoir indiqué les travaux sur les processus indexés par des arbres. Ce travail est également soutenu par le G.R. CNRS G0134 : "Traitement du Signal et Image". 
886I วมquəวəِ

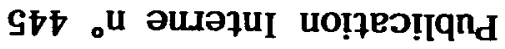

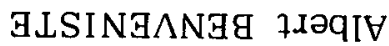

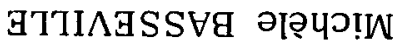

DNISSGDOYd TVNDIS

TVDILSILVIS GTVDSILTกW 


\section{Introduction.}

Recently, physicists and mathematicians ([4],[5]) proposed a new family of transforms, called wavelet transforms, which provide a time and scale decomposition of signals. More precisely, I. Daubechies [5] exhibits smooth functions $\phi$ such that the transform

$$
\hat{f}\left(2^{-n}, m\right)
$$

where

$$
\hat{f}(a, b)=\int_{-\infty}^{\infty} f(x) \frac{1}{\sqrt{a}} \phi\left(\frac{x-b}{a}\right) d x,
$$

provides a decomposition of $f$ on an orthonormal basis of $L^{2}(R)$. Notice that the test function $\phi$ is shifted for $n$ fixed and $m$ varying, and stretched for $m$ fixed and $n$ varying; hence scalar products with the shifted and stretched test function provide a time-and-scale decomposition of the signal $f$. Moving from a scale to the next finer one is done by associating to the point $\left(2^{-n}, m\right)$ the two points $\left(2^{-(n+1)}, 2 m\right)$ and $\left(2^{-(n+1)}, 2 m+1\right)$. From this elementary remark follows that the natural index set for $\hat{f}$ is the set of the vertices of the homogeneous dyadic tree.

Stationary processes on the homogeneous dyadic tree have been introduced in [1], where a Bochner-like theorem is stated which relates the covariance function of such processes to some spectral measure on the interval $[-1,+1]$. In [3], [6], [1], [2], dyadic trees are viewed as infinite, connected, non directed, acyclic graphs with 3 branches originating from each vertex, see figure 1. The purpose of this work is twofold. First, we need to introduce some convenient partial order on the dyadic tree to account for the desired multiscale interpretation we wish to introduce; second, we want to develop parametric models for such processes.

\section{Stationary processes on the dyadic tree.}

For the sake of simplicity, we shall denote by $\mathcal{T}$ the dyadic tree as well as the set of its vertices. Given two vertices $s$ and $t$, we denote by $d(s, t)$ the number of branches of the cycle free path linking these two vertices: $d$ is a distance on $\mathcal{T}$, and the two names of isometry or automorphism will both refer to any one-to-one map from $\mathcal{T}$ onto itself which preserves the distance $d$.

A process $\left(y_{t}\right)_{t \in \mathcal{T}}$ indexed by the dyadic tree $\mathcal{T}$ is said to be (second order) stationary if there exists a function $r$ from $N$ into $\mathbb{R}$ such that :

$$
\mathbb{E}\left(y_{t} y_{s}\right)=r(d(t, s))
$$


As expected, white noise corresponds to $r(n)=\delta(n)$ where $\delta$ denotes the Dirac function. White noise will be denoted by $\left(W_{t}\right)_{t \in \mathcal{T}}$ or simply $W_{t}$ in the sequel.

For $t_{1}, t_{2}, t_{3}$ shown in figure 1 , setting

$$
X=\left(y_{t_{1}} y_{t_{2}} y_{t_{3}}\right)
$$

its covariance matrix

$$
\mathbb{E}\left(X^{T} X\right)=\left(\begin{array}{ccc}
r_{0} & r_{2} & r_{2} \\
r_{2} & r_{0} & r_{2} \\
r_{2} & r_{2} & r_{0}
\end{array}\right)
$$

does not belong to the usual class of Toeplitz matrices characterizing covariance matrices of classical time series. Hence it is expected that Bochner's theorem relating the covariance function of a time serie to its spectral measure via a Fourier transform should be modified to account for guarantying the positivity of matrices such as the one above. The reader is referred to [1], [2], [7] for a statement of the Arnaud-Letac theorem which relates the covariance sequence of a stationary process on the dyadic tree to its spectral measure via a new transform.

\subsection{A partial order on the tree and its multiscale interpretation.}

Select an arbitrary extremity of the tree as pointed in the figure 1, and call it $-\infty$; then grasp the tree on $-\infty$, lift it and shake it: other extremities of the tree, being subject to gravity, are going to hang down as shown in figure 2 . Then the tree is shown to be partitioned according to horocycles, i.e. subsets of vertices lying at the same level on figure 2 (vertices on the same horocycle are at the "same distance" from $-\infty$ ). This informal transformation of the tree can be properly formalized as in [3], [7]. According to the discussion in the introduction about the wavelet transform, vertices on the same level have to be interpreted as sample points at a given level of resolution. Going down is interpreted as refining the scale, going up is moving to coarser scales. We shall write respectively

$$
s \preceq t(s \prec t)
$$

to indicate that $s$ lies above of or at the same level as $t$ (resp. strictly above $t$ ).

Now our next objective will be to introduce the counterpart of the usual shift operator $z^{-1}$ on sequences: here we need to encode moves on the tree $\mathcal{T}$. To encode such moves, we introduce the following 5 shift operators : 0 (no 
move), $\gamma^{-1}$ (move 1 step towards $-\infty$ ), $\alpha$ (move away from $-\infty$ by turning right), $\beta$ (move away from $-\infty$ by turning left), $\delta$ (exchanging closest neighbours at the same level), as depicted on figure 2 . These operators satisfy the following relations:

$$
\begin{aligned}
& \gamma^{-1} \alpha=0 \\
& \gamma^{-1} \beta=0 \\
& \gamma^{-1} \delta=\gamma^{-1} \\
& \delta^{2}=0 \\
& \delta \beta=\alpha
\end{aligned}
$$

Arbitrary moves on the tree can then be encoded via finite strings or words among the above alphabet after simplifications with suitable uses of the formulas above (exercise); such words will be generically denoted by $w, v, \ldots$, and the set of all such words will be denoted by $\mathcal{L}$. The length of a word $w$ is the length of the move according to the distance $d$; it is denoted by $|w|$ and is equal to the length of the string $w$ according to the rules

$$
\left\{\begin{array}{l}
\left|\gamma^{-1}\right|=|\alpha|=|\beta|=1 \\
|\delta|=2
\end{array}\right.
$$

If the word $w$ encodes a move towards a higher or identical (resp. strictly higher) level, we shall write

$$
w \preceq 0(w \prec 0)
$$

Examples (see figure 2):

$$
\begin{aligned}
& s_{1}=\gamma^{-4} t \\
& s_{2}=\delta \gamma^{-3} t \\
& s_{3}=\alpha \delta \gamma^{-3} t \\
& s_{4}=\alpha \beta \delta \gamma^{-3} t \\
& s_{5}=\beta^{2} \alpha \delta \gamma^{-3} t
\end{aligned}
$$

To shorten the notations, we introduce the operator $\delta^{(n)}$ defined recursively by :

$$
\begin{aligned}
& \text { if } t=\alpha \gamma^{-1} t \text { then } \delta^{(n)} t=\alpha \delta^{(n-1)} \gamma^{-1} t \\
& \text { if } t=\beta \gamma^{-1} t \text { then } \delta^{(n)} t=\beta \delta^{(n-1)} \gamma^{-1} t
\end{aligned}
$$

with $\delta^{(1)}=\delta$. Notice that $\left|\delta^{(n)}\right|=2 n$. For example, $s_{6}=\delta^{(4)} t$. 


\subsection{Spectral factorization and AR processes.}

We wish to decompose stationary processes $\left(Y_{t}\right)_{t \in \mathcal{T}}$ on the dyadic tree as follows

$$
Y_{t}=\sum_{s \preceq t} c_{t, s} W_{s}
$$

in a way similar to the usual Wold decomposition

$$
Y_{n}=\sum_{k \geq 0} c_{k} W_{n-k}
$$

for time series. Of particular interest will be the AR processes of order $n$ which satisfy a recursion of the form

$$
Y_{t}=\sum_{w \preceq 0,|w| \leq n} a_{w} Y_{w t}+\sigma W_{t}
$$

The two next surprising properties indicate how strange is the situation for AR processes. First, it is shown in [7] that the only stationary processes of the form

$$
Y_{t}=\sum_{s \in]-\infty, t]} a_{d(t, s)} W_{s}
$$

where $]-\infty, t$ ] denotes the path linking $t$ to $-\infty$, are the $\operatorname{AR}(1)$ processes! Second, it is also shown there that, in the above representation of $\operatorname{AR}(n)$ processes, $0\left(2^{n}\right)$ AR coefficients $a_{w}$ take place, whereas there are exactly $n$ degrees of freedom. To summarize, an exponentially growing number of coefficients are involved with an exponentially growing number of polynomial constraints: this naive representation of AR processes has to be dropped into trash. This problem will be the main subject of this paper.

\section{A Schur-Levinson parametrization.}

We shall show that the convenient solution of the above discussed problem about the parametrization of AR processes is provided by parametrizations of Schur-Levinson type. It is surprising that, unlike to time series, such parametrizations are not only alternative to other canonical parametrizations for AR processes, but rather are the only convenient ones!

\subsection{Innovations and residuals.}

Consider a stationary process $\left(Y_{t}\right)_{t \in \mathcal{T}}$ on the dyadic tree. In the sequel, we shall denote by $\mathcal{H}\{\cdots\}$ the Gaussian linear space spaned by the variables referred 
to in the brackets. Introduce the following family of spaces

$$
\mathcal{Y}_{t, n} \triangleq \mathcal{H}\left\{Y_{w t}: w \preceq 0,|w| \leq n\right\}
$$

$\mathcal{Y}_{t, n}$ denotes the "past" of $t$ of length $n$; notice that this space involves in particular indices which are at the same level as $t$. Notice also that, for $n=$ $2 m$, new vertices on the same level are added, whereas none are added for $n=2 m+1$.

Backward residual spaces $\left(\mathcal{F}_{t, n}\right)$ are defined by

$$
\mathcal{Y}_{t, n}=\mathcal{Y}_{t, n-1} \oplus \mathcal{F}_{t, n}
$$

The following backward residuals provide a basis for $\mathcal{Y}_{t, n}$ :

$$
F_{t, n}(w) \triangleq Y_{w t}-\mathbb{E}\left(Y_{w t} \mid \mathcal{Y}_{t, n-1}\right)
$$

where $w$ ranges over the words of $\mathcal{L}$ such that $|w|=n$ and $w \preceq 0$ (vertices in the "present/past" which are exactly at distance $n$ from $t$ ). Finally, we introduce

$$
F_{t, n} \triangleq \operatorname{col}\left(F_{t, n}(w)\right)
$$

where we refer the reader to [7] for a precise description of the ordering chosen on the $w$ 's to build this column vector. Notice that $\operatorname{dim} F_{t, n}=2^{\left[\frac{n}{2}\right]}$ where $[x]$ denotes the largest integer $\leq x$. Let us introduce now the forward residual spaces defined by

$$
\mathcal{Y}_{t, n}=\mathcal{Y}_{\gamma^{-1} t, n-1} \oplus \mathcal{E}_{t, n}
$$

The forward residuals

$$
E_{t, n}(w) \triangleq Y_{w t}-\mathbb{E}\left(Y_{w t} \mid \mathcal{Y}_{\gamma^{-1} t, n-1}\right)
$$

where $w$ ranges over the words of $\mathcal{L}$ such that $|w| \leq n$ and $w \asymp 0$ (vertices at distance $<n$ on the same level as $t$ ) provide a basis of the forward residual spaces. Setting

$$
E_{t, n} \triangleq \operatorname{col}\left(E_{t, n}(w)\right)
$$

we have $\operatorname{dim} E_{t, n}=2^{\left[\frac{n-1}{2}\right]}$.

\subsection{Normalized Levinson recursions and whitening filters.}

In the sequel, if $X$ is a vector random variable, we shall denote by $X^{*}$ the same variable normalized by the inverse symmetric square root of its covariance 
matrix. We shall also need the following notations. Introduce

$$
\mathbb{1}_{*}=\frac{1}{\sqrt{\operatorname{dim} \mathbb{1}}} \mathbb{1}, \cup_{*}=\mathbb{1}_{*} \mathbb{1}_{*}^{T}
$$

Then $U_{*}$ possesses $(1,0, \ldots, 0)$ as eigenvalues. We introduce also the following identities and definitions :

$$
\begin{aligned}
\cup_{*} \cup_{*} & =\cup_{*} \\
f^{*} & =\mathbb{1}_{*}^{T} F^{*}=\frac{1}{\sqrt{\operatorname{dim} F}} \sum_{w} F^{*}(w) \\
\mathbb{1}_{*} f^{*} & =\cup_{*} F^{*}
\end{aligned}
$$

Theorem 1: the normalized residuals $E_{t, n}^{*}, F_{t, n}^{*}$ obey the following recursions :

n even :

$$
\left\{\begin{array}{l}
E_{t, n}^{*}=\Sigma_{E, n}^{*-\frac{1}{2}}\left[E_{t, n-1}^{*}-k_{n} \mathbb{1}_{*} f_{\gamma^{-1} t, n-1}^{*}\right] \\
F_{t, n}^{*}=\Sigma_{F, n}^{*-\frac{1}{2}}\left[\left(\begin{array}{c}
E_{\delta\left(\frac{n}{2}\right)_{t, n-1}}^{*} \\
F_{\gamma^{-1} t, n-1}^{*}
\end{array}\right)-\sqrt{2} k_{n} \mathbb{1}_{*} e_{t, n-1}^{*}\right]
\end{array}\right.
$$

where

$$
\begin{aligned}
\Sigma_{E, n}^{*} & \triangleq \Sigma_{E, n-1}^{\frac{-1}{2}} \Sigma_{E, n}^{\frac{1}{2}} \\
& =I-k_{n}^{2} U_{*}
\end{aligned}
$$

and

$$
\Sigma_{F, n}^{*}=\left(\begin{array}{cc}
I-k_{n}^{2} \cup_{*} & \left(k_{n}-k_{n}^{2}\right) \cup_{*} \\
\left(k_{n}-k_{n}^{2}\right) \bigcup_{*} & I-k_{n}^{2} \bigcup_{*}
\end{array}\right)
$$

n odd : First for $n=1$ the residuals are scalar and are given by

$$
\left\{\begin{array}{l}
e_{t, 1}^{*}=\frac{1}{\sqrt{1-k_{1}^{2}}}\left(e_{t, 0}^{*}-k_{1} f_{\gamma^{-1} t, 0}^{*}\right) \\
f_{t, 1}^{*}=\frac{1}{\sqrt{1-k_{1}^{2}}}\left(f_{\gamma^{-1} t, 0}^{*}-k_{1} e_{t, 0}^{*}\right)
\end{array}\right.
$$

then, for $n>1$ setting

$$
h_{n-1} \triangleq \operatorname{cor}\left(e_{t, n-1}^{*}, e_{\delta\left(\frac{n-1}{2}\right)}^{*}\right)=\frac{k_{n-1}}{1+k_{n-1}}
$$


we have :

$$
\begin{aligned}
& E_{t, n}^{*}=\sum_{E, n}^{*-1 / 2}\left[\left(\begin{array}{l}
E_{t, n-1}^{*} \\
E_{\delta^{\left(\frac{n-1}{2}\right)}}^{*} t, n-1
\end{array}\right)-k_{n} \sqrt{1+h_{n-1} \mathbb{1}_{*}} f_{\gamma^{-1} t, n-1}^{*}\right] \\
& F_{t, n}^{*}=\sum_{F, n}^{*-1 / 2}\left[F_{\gamma^{-1} t, n-1}^{*}-l_{n} \mathbb{1}_{*} \frac{1}{2}\left(e_{t, n-1}^{*}+e_{\delta^{\left(\frac{n-1}{2}\right)}}^{*}\right)\right]
\end{aligned}
$$

where $l_{n}=k_{n} \sqrt{\frac{2}{1+h_{n-1}}}$

$$
\Sigma_{E, n}^{*}=\left(\begin{array}{cc}
I-k_{n}^{2} \frac{1+h_{n-1}}{2} \cup_{*} & \left(h_{n-1}-k_{n}^{2} \frac{1+h_{n-1}}{2}\right) \cup_{*} \\
\left(h_{n-1}-k_{n}^{2} \frac{1+h_{n-1}}{2}\right) \cup_{*} & I-k_{n}^{2} \frac{1+h_{n-1}}{2} \cup_{*}
\end{array}\right)
$$

and

$$
\begin{aligned}
\Sigma_{F, n}^{*} & \triangleq \Sigma_{F, n-1}^{\frac{-1}{2}} \Sigma_{F, n}^{\frac{1}{2}} \\
& =I-k_{n}^{2} \bigcup_{*}
\end{aligned}
$$

According to these formulas, it turns out that the $n$-th order residuals are entirely determined by exactly $n$ reflection coefficients $k_{1}, \ldots, k_{n}$, although the $n$-th order residual spaces are of dimension approximately equal to $2^{\left[\frac{n}{2}\right]}$. This very surprising fact is a consequence of the very large amount of symmetries that are found within the tree $\mathcal{T}$ as suggested by the way this tree is drawn in the figure 1 . On the other hand, the reflection coefficients are easily computed using scalar recursions involving scalar barycentric residuals only. More precisely, setting

$$
\begin{aligned}
& e_{t, n}=2^{-\left[\frac{n-1}{2}\right]} \sum_{|w|<n, w \asymp 0} E_{t, n}(w) \\
& f_{t, n}=2^{-\left[\frac{n}{2}\right]} \sum_{|w|=n, w \preceq 0} F_{t, n}(w)
\end{aligned}
$$

we get the

\section{Theorem 2: barycentric Levinson recursions.}

n even

$$
\left\{\begin{array}{l}
e_{t, n}=e_{t, n-1}-k_{n} f_{\gamma^{-1} t, n-1} \\
f_{t, n}=\frac{1}{2}\left(f_{\gamma^{-1} t, n-1}+e_{\delta^{\left(\frac{n}{2}\right)} t, n-1}\right)-k_{n} e_{t, n-1}
\end{array}\right.
$$


where

$$
\begin{aligned}
k_{n} & =\operatorname{cor}\left(e_{t, n-1}, f_{\gamma^{-1} t, n-1}\right) \\
& \left.=\operatorname{cor}\left(e_{\delta^{\left(\frac{n}{2}\right)}}\right)_{t, n-1}, e_{t, n-1}\right) \\
& \left.=\operatorname{cor}\left(e_{\delta^{\left(\frac{n}{2}\right)}}\right)_{t, n-1}, f_{\gamma^{-1} t, n-1}\right)
\end{aligned}
$$

moreover

$$
\mathbb{E}\left(e_{t, n-1}^{2}\right)=\mathbb{E}\left(e_{\delta^{\left(\frac{n}{2}\right)_{t, n-1}}}^{2}\right)=\mathbb{E}\left(f_{\gamma^{-1} t, n-1}^{2}\right) \triangleq \sigma_{n-1}^{2}
$$

and

$$
\left\{\begin{array}{l}
\sigma_{e, n}^{2}=\left(1-k_{n}^{2}\right) \sigma_{n-1}^{2} \\
\sigma_{f, n}^{2}=\left(\frac{1+k_{n}}{2}-k_{n}^{2}\right) \sigma_{n-1}^{2}
\end{array}\right.
$$

so that the following constraint must be satisfied by the reflection coefficient in order to avoid singular residuals for $n$ even :

$$
-\frac{1}{2}<k_{n}<1
$$

n odd for $n>1$

$$
\left\{\begin{array}{l}
e_{t, n}=\frac{1}{2}\left(e_{t, n-1}+e_{\left.\delta^{\left(\frac{n-1}{2}\right.}\right)_{t, n-1}}\right)-k_{n} f_{\gamma^{-1} t, n-1} \\
f_{t, n}=f_{\gamma^{-1} t, n-1}-\frac{1}{2} k_{n}\left(e_{t, n-1}+e_{\left.\delta^{\left(\frac{n-1}{2}\right.}\right)_{t, n-1}}\right)
\end{array}\right.
$$

where

$$
k_{n}=\operatorname{cor}\left(\frac{1}{2}\left(e_{t, n-1}+e_{\delta^{\left(\frac{n-1}{2}\right)} t, n-1}\right), f_{\gamma^{-1} t, n-1}\right)
$$

Moreover

$$
\mathbb{E}\left(e_{t, n}^{2}\right)=\mathbb{E}\left(f_{\gamma^{-1} t, n}^{2}\right) \triangleq \sigma_{n}^{2}
$$

and

$$
\sigma_{n}^{2}=\left(1-k_{n}^{2}\right) \sigma_{f, n-1}^{2}
$$

so that the following condition must hold for $n$ odd:

$$
-1<k_{n}<1
$$

For $n=1$, the combination of the two forward residuals is replaced by the single one $e_{t, 0}$.

MAIN REMARK: Notice the fundamental difference with Levinson recursions for time series: here for $n$ even, we must have $-1 / 2<k_{n}$. 


\subsection{Further results.}

Apart from the proofs of the two theorems above, the following further results can be found in [7].

First, modelling filters are derived from the whitening filters of theorem 1 . $\operatorname{AR}(\mathrm{n})$ processes are characterized by the property that, as expected, $k_{n+m}=0$ for $m>0$; it is also shown that $\operatorname{AR}(\mathrm{n})$ processes are exactly the processes which possess an impulse response with support contained in the cylinder with axis $\left(\gamma^{-k}\right)_{k \geq 0}$ and radius $n-1$, thus extending the result we have given for $n=1$.

Second, it is shown that the above conditions on reflection coefficients are sufficient to guaranty the existence of an associated finite order AR process, and to guaranty that the corresponding modelling filters are stable when $t$ moves away from $-\infty$.

Third, Schur recursions on formal power series are given that are the counterpart of the Schur recursions for ordinary time series. By the way, we provide an effective criterion to check whether a sequence $\left(r_{n}\right)_{n \geq 0}$ is the covariance sequence of a stationary process on the dyadic tree (Schur criterion).

\section{Conclusion.}

A new framework for multiscale statistical signal processing has been introduced. Its purpose is to provide a statistical toolbox to analyse properties of signals involving simultaneously time and scale. Stationary processes over the dyadic tree were borrowed from harmonic analysts for this purpose, and a new partial order was proposed to model causality in scale. AR processes were investigated and it has been shown that Schur-Levinson parametrizations play a crucial role. Finally, as expected from the introduced model, the restriction at a given scale (level) of a sample of such processes looks like a fractal, i.e. a random signal appearing similar whenever seen from either close or far away. For instance the figure 3 shows such a sample for an AR(1) process with $k_{1}=0.99$.

\section{References}


[2] J.P. ARNAUD : "Fonctions sphériques et fonctions définies positives sur l'arbre homogène". C.R.A.S. t.290, série A (14 Janv. 1980), p.99101.

[3] P. CARTIER : "Géométrie et analyse sur les arbres". Séminaire Bourbaki, n407, Février 1972.

[4] I. Daubechies, A. Grossmann, Y. Meyer : "Painless non-orthogonal expansions". Jal Math. Phys., vol.27, p.1271-1283, 1986.

[5] I. DAUBECHIES : "Orthonormal bases of compactly supported wavelets". Res. Rep. AT and T Bell Labs., 1987. to appear in Comm. on Pure and Appl. Math.

[6] J.P. Arnaud, J.L. Dunau, G. Letac: "Etude d'une classe de marches aléatoires sur l'arbre homogène" dans "Arbres homogènes et Couples de Gelfand", Publications du Laboratoire de Statistique et Probabilités, Univ. Paul Sabatier, Toulouse, no02-83, Juin 1983.

[7] M. Basseville, A.Benveniste: "Modèles statistiques temps-échelle en traitement du signal", IRISA res. rep. 1989. 

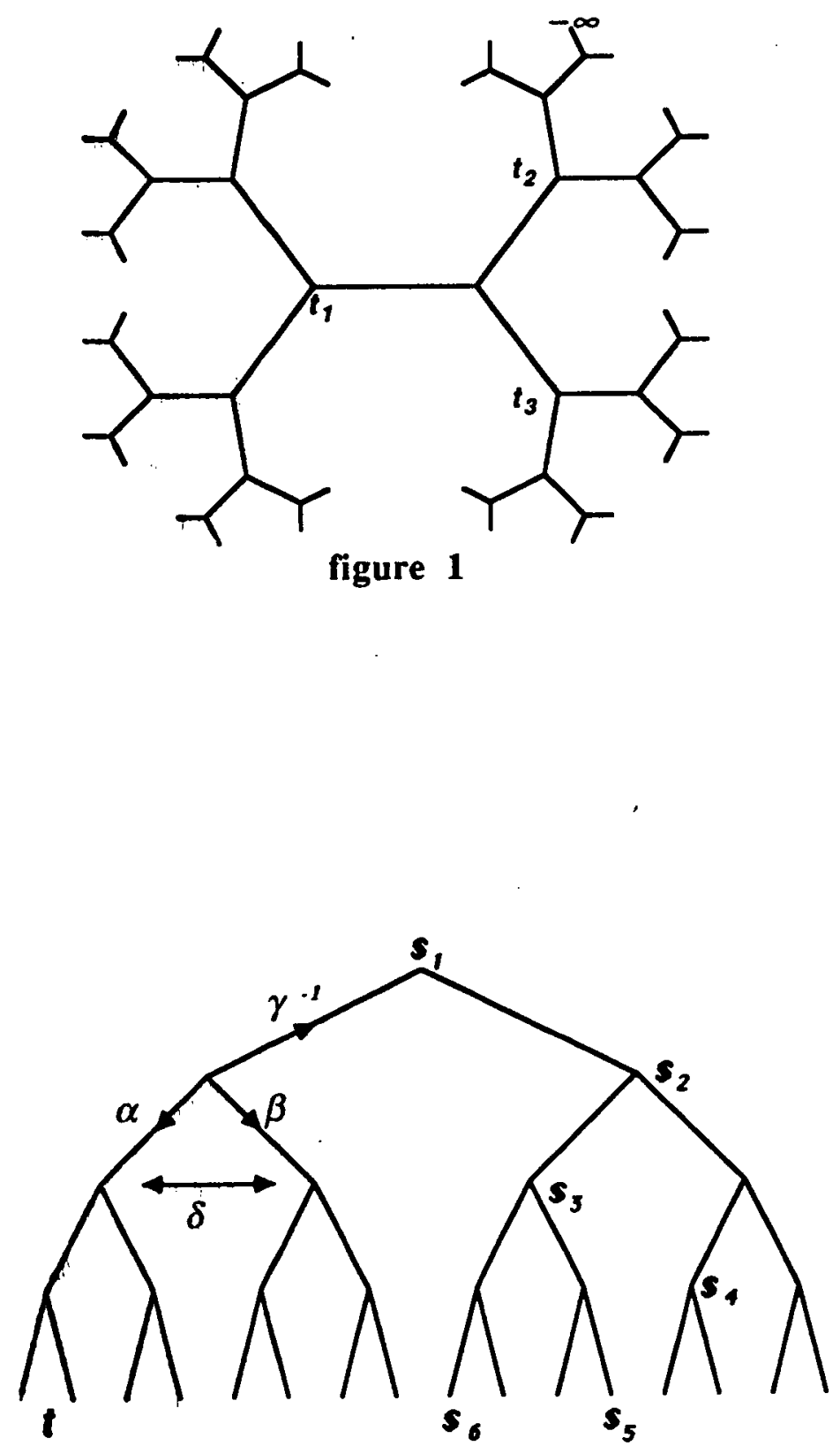

figure 2 
figure 3

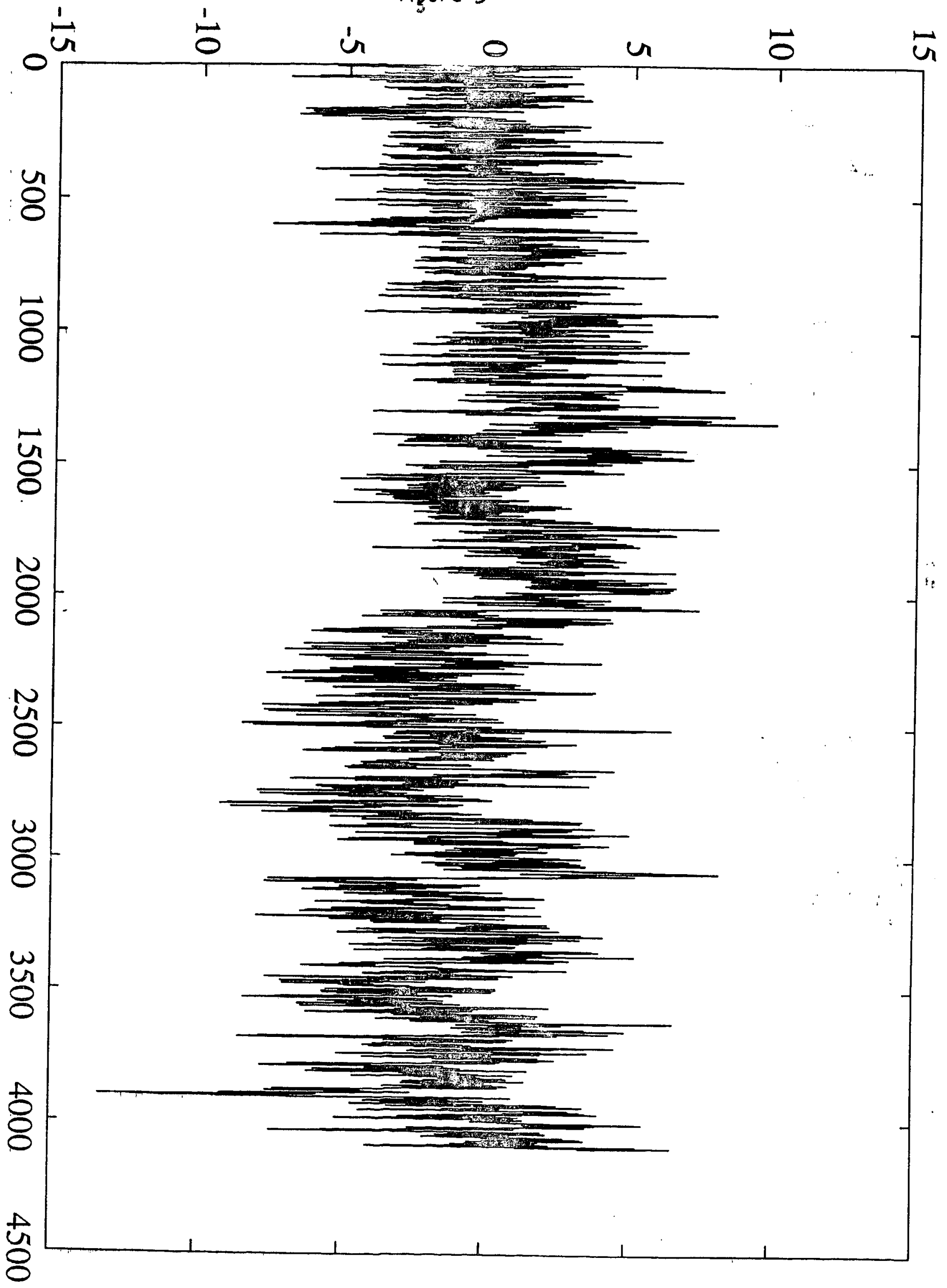


PI 438 - A PROPOS DE LA RESOLUTION D'UN SYSTEME LINEAIRE DANS UN CORPS FINI : ALGORITHMES ET MACHINES PARALLELES

Hervé LE VERGE, Patrice QUINTON, Yves ROBERT, Gilles VILLARD 22 Pages, Novembre 1988.

PI 439 - ALPHA DU CENTAUR : A PROTOTYPE ENVIRONMENT FOR THE DESIGN OF PARALLEL REGULAR ALGORITHMS

Pierrick GACHET, Patrice QUINTON, Christophe MAURAS, Yannick SAOUTER

20 Pages, Novembre 1988.

PI 440 - CONSTRUCTION METHODIQUE D'UN ALGORITHME REPARTI DE DETECTION DE LA TERMINAISON

Jean-Michel HELARY, Michel RAYNAL

18 Pages, Décembre 1988.

PI 441 - LES GRAPHES A MOTIF

Didier CAUCAL

46 Pages, Décembre 1988.

PI 442 - CAUSAL TREES

Philippe DARONDEAU, Pierpaolo DEGANO

44 Pages, Décembre 1988.

PI 443 - TROIS IMPLANTATIONS DU RECUPERATEUR DE MEMOIRE DE LA MACHINE MALI

Michel LE HENAFF, Hervé SANSON

118 Pages, Décembre 1988.

PI 444 - ANALYSE FACTORIELLE LISSEE ET ANALYSE FACTORIELLE DES DIFFERENCES LOCALES

Brigitte ESCOFIER, Habib BENALI

- 34 Pages, Décembre 1988.

PI 445 - MULTISCALE STATISTICAL SIGNAL PROCESSING

Michèle BASSEVILLE, Albert BENVENISTE

16 Pages, Décembre 1988. 
.

i. 